\title{
Association of genetic variation with disproportionate vitamin A deficiency in pregnant women of certain ethnicities in the United States.
}

Masako Suzuki ( $\square$ masako.suzuki@einsteinmed.org )

Albert Einstein College of Medicine https://orcid.org/0000-0003-0605-9225

Tao Wang

Albert Einstein College of Medicine

Diana J Garretto

Stony Brook University Hospital

Carmen R. Isasi

Albert Einstein College of Medicine

Wellington V Cardoso

Columbia University

John M. Greally

Albert Einstein College of Medicine

Loredana Quadro

Rutgers University

Short report

Keywords: Vitamin A deficiency, pregnant women, Hispanics, NHANES

Posted Date: November 18th, 2020

DOI: https://doi.org/10.21203/rs.3.rs-49188/v2

License: (1) This work is licensed under a Creative Commons Attribution 4.0 International License.

Read Full License 


\section{Abstract}

Vitamin A is an essential micronutrient that plays critical roles in many biological functions of the body. Limited access to vitamin A-rich food or supplements severely affects tissue and blood levels of vitamin A. Therefore, low serum vitamin A and poverty levels are strongly associated in vitamin A deficiency (VAD) studies that have focused mainly on developing countries. The current national prevalence rate of vitamin A deficiency in the United States is reported to be very low $(<1 \%)$. However, several studies, including ours, have suggested that people from certain ethnic groups still face a higher proportion of vitamin A deficiency. We hypothesize that the genetic variations between ethnic groups may associate to the VAD proportional differences between women of different ancestries. To assess the associations, we reanalyzed two independent datasets of serum retinol levels of pregnant women in the United States and three datasets of genotypic information of different ancestries. We found that pregnant women with nonHispanic Black and with Latin American/Afro-Caribbean ancestry have strikingly high proportions of VAD compared to non-Hispanic White and Latin American/Mexican ancestry. Genotypic analyses showed that the minor allele frequencis of genetic variants that associate to serum retinol levels have significantly higher variations between these different ancestries. Our study revealed that VAD rates in the pregnant women differ between different ancestries and that ancestry-dependent genetic variations might contribute to the differences.

\section{Main Text}

The essential micronutrient vitamin A plays critical roles in vision [1, 2], immune system [3, 4], cell growth and differentiation [5-8], as well as in the development of multiple organs, including the lung, heart, eyes, and kidneys (reviewed in $[9,10]$ ). Since vitamin A is an essential micronutrient, limited access to vitamin Arich food or supplements severely affects tissue and blood levels of vitamin $A$ in humans [11]. In the United States (U.S.), while the current prevalence rate of vitamin A deficiency (VAD) - defined as serum retinol levels lower than $1.05 \mu \mathrm{mol} / \mathrm{l}$ - is estimated to be less than 1\% (CDC's Second Nutrition Report [12]), it varies between different ethnic groups and races [13]. By using datasets from the National Health and Nutrition Examination Survey (NHANES) that measured vitamin A (retinol and its esterified form, retinyl ester) and provitamin A carotenoids in the serum of over 24,000 individuals in 2001-2002, 2003-2004, and 2005-2006 [14], we found that the sample-weighted serum retinol levels of vitamin A ranged from 1.74 (non-Hispanic Black) to $2.09 \mu \mathrm{mol} / \mathrm{L}$ (non-Hispanic White) (Additional file 1). For this analysis, we employed sample weights as recommended by the CDC to produce an unbiased national estimates [14]. Moreover, an epidemiological study based on the NHANES datasets demonstrated that non-Hispanic Black and Mexican American women of childbearing age have a higher rate of VAD [15]. However, since the overall prevalence of VAD is considered to be rare in developed countries, vitamin A levels have not been measured routinely and populations levels of the vitamin are not up to date [16]. Therefore, the sparse available data have failed to detect the up-to-date status of VAD for individuals in developed countries. 
Recently, we reported a high VAD proportion rate among pregnant women in the Bronx, NY, USA [17], where the ethnic diversity and the poverty rate is much higher than in the rest of the nation [12]. The original study addressed the effects of bariatric surgery on serum vitamin A levels during pregnancy [17]. The most surprising result we observed in the study was that more than $60 \%$ of the pregnant women who did not undergo the bariatric surgery (control group) had serum retinol levels lower than $1.05 \mu \mathrm{mol} / \mathrm{I}$ [17], i.e., they were vitamin A deficient. The proportion of vitamin A deficient women in the Bronx was much higher than that of non-Hispanic Black and Hispanic (Mexican American) women of the same age group reported by Hanson et al. upon analysis the NHANES dataset [15]. This finding prompted us to test the disproportionality of VAD status in pregnant women between different ethnic groups by re-analyzing our data from the Bronx study [17] as well as those from NHANES [18]. While the NHANES data were collected almost two decades ago, this is the latest and most current nationwide serum vitamin A level assessment in the U.S.. We hypothesized that the proportion of pregnant women with VAD differs between different ancestry groups, and that ancestry-dependent genetic variations could contribute to the variations. To test this possibility, we re-analyzed the two independent serum retinol datasets of pregnant women in the U. S. mentioned above and three genetic variation information.

We evaluated serum retinol levels, the measure of VAD status [11, 19-21], identifying distinct ancestries of participants in two separate datasets, and tested the possibility that genetic variation could contribute to these disproportionalities using three publicly available allele frequency databases. Firstly, we reanalyzed the third trimester serum retinol levels in the Bronx dataset by ethnic group, specifically using the self-reported ethnicities (non-Hispanic Black, Hispanic, or other race) as the ethnicities [17]. In the Bronx dataset, women were approached in the antepartum period during prenatal care for recruitment and written informed consent was obtained. Women planning to breasfeed with singleton pregnancy and no history of malabsorption were recruited in the control group. The bariatric surgery group included women that were planning to breasfeed with singleton pregnancy and a history of bariatric surgery (either Rouxen-Y or gastric sleeve) [17]. Demographic data included race/ethnicity, education, pre-pregnancy and at delivery body mass index, gestational weight gain and parity. Pregnancy outcome included gestational age at delivery, mode of delivery, and neonatal weight [17]. Secondly, we analyzed the pregnant serum retinol levels by ethnicities using the reported race/ethnicity (RIDRETH1) information in the Sample Person Demographics Files from the NHANES dataset [14]. To identify the genetic variants which might be associated with the differences in the proportions of VAD between ethnic groups, we tested the deviations of allele frequency between the groups. The allele frequency of the variants of each ethnic group were obtained from Allele Frequency Aggregator (ALFA) [22], the Population Architecture using Genomics and Epidemiology [PAGE [23], BioProject Accession: PRJNA168052], and 1000 Genomes project [24]. The ALFA pipeline consists of the allele frequency for variants in $\mathrm{dbGaP}$ that incldes genomic data with subjects from 12 diverse populations worldwide [22]. The PAGE consortium dataset includes genetic variations representing seven ethnic groups, i.e., African Americans, Asian Americans, American Indians, European Americans, Hispanic Americans, and Native Hawaiians, from the United States-based cohorts [23]. The Hispanic American cohorts in PAGE contains subjects from the Hispanic Community Health Study/Study of Latinos (HCHS/SOL), with one of the HCHS/SOL research centers located in the Bronx, NY [23, 25]. The associations of genetic variants with gene expression levels, defining expression quantitative trait loci 
(eQTL), were obtained from the Genotype-Tissue Expression project, GTEx [26]. We used Student's t-test for continuous variables and Fisher's exact test for categorical variables. All statistical analyses were performed using R version 4.0.2 [27].

From the Bronx study, we only used the third-trimester serum retinol levels that were available for 67 women out of the 96 participants [17]. We found no significant association between missing data status and other covariates. While maternal serum levels of beta-carotene, the most abundant dietary vitamin $A$ precursor [11], and cord blood serum retinol were found to be significantly associated with maternal VAD status ( $p=0.041$ and $p=0.007$, respectively), other known covariates, including the bariatric surgery status, did not show significant associations (Table 1). The proportion of VAD in Hispanic women was $65.9 \%$ (29 out of 44 Hispanic participants), in non-Hispanic Blacks was 53.3\% (8 of 15 African American participants), and for other ethnicities was 37.5\% (3 in 8 participants). Among the Hispanic participants $(n=44)$, vitamin A deficient women tended to be younger than the vitamin A sufficient women $(p=0.088)$, but education levels, pre-pregnancy body mass index (BMI), and gestational weight gain (GWG) were not associated with the VAD status ( $p=0.876, p=0.195$, and $p=0.935$, respectively). Whereas the poverty levels of each cohort were not assessed in the Bronx study [17], the degree of education and poverty level are generally negatively correlated in the Bronx, NY (poverty rate of less than High School is $36.6 \%$, High School is 23.4\%, and College is 19.5\%, 20191 -year estimates, U.S. Census) [28]. The U.S. Census Bureau determines poverty status if the household income is lower than the official poverty measure threshold (below the poverty income ratio (PIR) 1.0), the minimum income needed to avoid poverty [28]. Therefore, we inferred that the poverty levels in the Bronx study were likely similar between the vitamin A deficient and sufficient women, and that the poverty level might not have been directly associated with VAD status between ethnicity, in this study. Also, no clinical evidence was available for these VAD pregnant females as they had not been assessed [17]. Nevertheless, overt symptoms of VAD were not expected given that their deficiency status should be considered subclinical, based on serum levels.

Despite the relatively limited sample size of the Bronx study, we found that the proportion of VAD in Hispanic women was higher in African American women and both proportions were much higher than the estimated levels in the U.S. (Additional file 1). In the NHANES dataset the proportion of VAD among Hispanic (Mexican American) pregnant women was half that of non-Hispanic Black pregnant women, $14.9 \%$ and $32.0 \%$ respectively ( $p<0.0001$, Fisher's exact test, Additional file 1; 966 pregnant women). Blumberg et al. reported that PIR $>1.85$ subgroup had a lower prevalence of inadequacy for most nutrients compared to the subgroups PIR $\leqq 1.85$ [29], and about $45 \%$ of people live below PIR $\leqq 1.85$ in the Bronx (U.S. Census [28]). Therefore, from this latter dataset, we focused our analysis on pregnant women aged 17-42 (similar to the age range of the Bronx study) with a PIR less than 1.85. While VAD status and $\mathrm{PIR} \leqq 1.85$ were significantly associated in non-pregnant and pregnant non-Hispanic Black women (aged 17-42, Fisher's exact test $\mathrm{p}<0.005,95$ percent confidence interval $(95 \% \mathrm{Cl})$ 1.37-4.45), this association was not observed in pregnant women of all races (Hispanic (Mexican American), Fisher's exact test $p=0.6494$ (95\% Cl 0.53-4.57); non-Hispanic Black, Fisher's exact test $p=0.12$ (95\%Cl 0.83-6.73); non-Hispanic White, $\mathrm{p}=0.684(95 \% \mathrm{Cl} 0.49-2.71)$. Taken together, the higher VAD rates among pregnant women between these 
minority groups (Hispanic and non-Hispanic Black) from the two datasets might be independent of their poverty levels.

Interestingly, the proportions of VAD in non-Hispanic Black pregnant women were not significantly differ between the datasets $(p=0.26,95 \% \mathrm{Cl} 0.14-1.74)$, however, the proportions of VAD in Hispanic (Mexican) pregnant women in poverty from the NHANES dataset were significantly lower than that of Hispanic pregnant women in the Bronx dataset $(p=1.973 \mathrm{e}-10,95 \% \mathrm{Cl} 0.04-0.22)$. The major origins of Hispanic populations in the Bronx are Latin Americans with Afro-Caribbean ancestry [39.4\% Dominicans and 36.4\% Puerto Ricans, the U.S. Census data [28]], whereas the Hispanic population in the NHANES data we used is Mexican Americans. Thus, we asked whether the ancestry-specific genetic variations might contribute to lower serum retinol levels. Genetic contributions to the levels of circulating retinol have been reported in European populations. Specifically, a family study in France showed that the heritability estimate for serum retinol concentration (30.5\%) was much larger than the variability accounted for by household, i.e., individuals living in the same house (14.2\%) [30]. Moreover, in the GWAS Catalog [31], two single nucleotide polymorphisms (SNPs) (rs10882272 T/C and rs1667255 C/A), which are located near the transthyretin (TTR) and retinol binding protein 4 (RBP4) genes, identified from a genome-wide association study of 5,006 "Caucasian" males, are listed as associated with serum retinol levels [32]. The association of rs10882272 was replicated in independent samples, including 3,792 females and 504 males [32]. However, the rate of VAD in the individuals studied was low, thus the authors were not able to test the association of the genetic variation with VAD [32]. To assess if this genetic variation could be associated with VAD status differences between racial/different ethnic groups, we compared the low serum retinol allele frequencies of rs 10882272 and rs 1667255 between different ethnic groups in publicly available datasets, namely ALFA and PAGE. While we did not observe significant differences in major allele frequencies of rs 1667255 between Hispanic groups (Additional file 2), as we predicted, the allele frequencies of rs 10882272 showed significant variation between different ethnic groups. The frequency of the allele associated with low serum retinol (rs10882272: frequency for $\mathrm{C}$ allele) was much higher in African $(0.620,62 \%)$ and African American (0.617) compared to European (0.383) and Asian (0.106) individuals in the ALFA dataset (Figure 1a). Similarly, the PAGE dataset results showed that the risk allele frequencies were higher in Latin Americans with Afro-Caribbean ancestry [Puerto Ricans (0.455), Dominicans (0.502) and Cubans (0.410)] compared to Mexicans (0.260), Central Americans (0.288), South Americans (0.278) or Native Americans (0.357) (Figure 1b). The ethnic groups with the higher VAD proportions showed a higher frequency of the allele associated with low serum retinol levels. The rs10882272 variant is located in the 3' UTR of the free fatty acid receptor 4 (FFAR4) gene and downstream of RBP4 gene. The FFAR4 gene encodes a GPCR receptor (GPCR120) for free long-chain fatty acids, including omega-3 [33, 34]. FFAR4/GPCR120 is expressed in various cell types, including pituitary, lung, macrophages, adipocytes, intestinal neuroendocrine cells and pancreatic cells. Thus, it participates in a number of physiological processes, including energy regulation, insulin sensitivity, immunological homeostasis and anti-inflammatory responses [35]. RBP4 is the sole specific carrier for retinol in the bloodstream $[2,36]$. Predominantly expressed in the hepatocytes, RBP4 binds retinol to mobilize vitamin A from the liver, the primary body storage site of the vitamin, towards the peripheral tissues [2]. We tested if the rs 10882272 is an expression quantitative trait locus (eQTL) for its nearby genes using a publicly 
available database (the Genotype-Tissue Expression project, GTEx) [26]. In the GTEx data, we detected the associations between the rs 10882272 variations and the expression levels of RBP4 in the liver, where the gene is highly expressed [37], with the presence of the allele associated with low serum retinol levels also associated with increased expression of $R B P 4$ (normalized effect size: $0.137, \mathrm{p}=0.00012$, and $\mathrm{m}$-value 0.987). For FFAR4, we detected the association in lung (normalized effect size: $0.126, \mathrm{p}=8.5 \mathrm{e}-6$, and $\mathrm{m}$ value 1.00), but not in pituitary (normalized effect size: $0.0334, p=0.5$, and m-value 0.809 ). The pituitary showed highest expression of FFAR4 in the GTEx data.

Another genetic variant associated with differences in serum retinol levels between different ethnic groups is the rs738409 polymorphism [38], which is located in the patatin-like phospholipase domain containing 3 (PNPLA3) gene. PNPLA3 encodes a gene involved in the mobilization of retinyl esters stored in stellate cells $[38,39]$. The rs 738409 polymorphism is a missense variant, the $C$ to $G$ nucleotide substitution changing the amino acid I[ATC] to M[ATG]. The PNPLA3 I148M missense variant is a loss-of-function mutation [38], and the associations between the variant and the risk of nonalcoholic fatty liver disease (NAFLD) has been reported [40-42]. The frequency of the mutant allele varies between ethnic groups (0.144 to 0.499, African American to Mexican; PAGE dataset). Of note, individuals homozygous for PNPLA3 I148M have lower circulating levels of RBP4 [38]. Changes in circulating levels of RBP4 have been linked to pathological conditions and variations in nutritional intake [43-46]. Interestingly, reported associations of the circulating levels of RBP4 and NAFLD are conflicting, and a recent meta analysis reported that circulating RBP4 levels may indeed not be associated with NAFLD [47]. Thus, we speculate that the associations between PNPLA3 I148M variants and circulating RBP4 levels might be independent of NAFLD status. In animal models, while retinol deficiency leads to accumulation of RBP4 in liver, likely by inhibiting its secretion from this organ, hepatic RBP4 mRNA levels show no differences between vitamin A deficiency and sufficiency [48]. Further studies are needed to test the associations of these SNPs with circulating RBP4 levels, serum retinol levels, and disease status.

Not only these three SNPs, but also several GWAS and candidate gene association studies have also identified other polymorphisms associated with serum retinol and beta-carotene levels and the betacarotene bioactivities [49]. We thus also assessed the allele frequency deviations of the 39 SNPs associated with circulating vitamin A levels [49] between different ethnic groups in the 1000 Genome Project [24]. The deviations of allele frequencies of those vitamin A-related SNPs between different ethnic groups are listed in the Additional file 2. The average of the allele frequency standard deviation among ethnic groups was 0.122 , significantly higher than randomly selected sets of 39 SNPs from the 1000 Genomes data ( $p=0.030$, permutation test with 1,000 iterations, Additional file 1$)$. Since serum retinol level variations between different ethnic groups have been reported, this result is not surprising. However, this is the first systematic analysis of the allele frequency variations of the vitamin A-related SNPs among different ethnic groups. Looker et al. reported serum retinol level differences among three Hispanic groups using the Hispanic Health and Nutrition Examination Survey (HHANES) conducted from 1982-1984 [50]. The authors found that Mexican Americans have a higher VAD prevalence rate than Puerto Ricans or Cubans in both adults and children. This study was performed almost four decades ago when the participants' nutrient status might have differed from the current one. The CDC's Second Nutrition Report 
in 2012 showed that serum vitamin A concentrations increased between 1999-2000 and 2005-2006 in the United States (geometric mean, $52.8 \mu \mathrm{g} / \mathrm{dl}$ to $54.7 \mu \mathrm{g} / \mathrm{dl}$ ). Moreover, serum retinyl palmitate levels, an indicator of newly ingested vitamin A, were dramatically increased during this period in Mexican Americans (geometric mean $0.759 \mathrm{ug} / \mathrm{dl}$ in $1999-2000$ to $1.85 \mathrm{ug} / \mathrm{dl}$ in 2005-2006) [51]. The differences observed in 1982-1984 might reflect the lower dietary intake of vitamin A at that time and not genetic variation. An updated population-based measurement of serum retinol levels is clearly needed.

We acknowledge that there are several limitations to our study: the sample size of the Bronx cohort $(n=97)$ was limited, the poverty levels and clinical information on VAD-related outcome of the Bronx cohort was not availale, the serum retinol data of NHANES were collected more than a decade ago, and the genotype information of all participants is not available in both cohorts. Further genome-wide association studies with demographic information, including food accessibility/intake in multiethnic cohorts, are needed to assess the influences of genetic variation and the different VAD status between different ethnic groups.

In summary, while VAD in developed countries is believed to be a rare condition, there is a substantial proportion of VAD pregnant women of certain ethnic groups, even in wealthy, developed countries. While the WHO does not recommend routine vitamin A supplementation to pregnant women, they recommend vitamin A supplementation to pregnant women in a given geographical area if $\geq 20 \%$ of pregnant women have serum retinol levels $<0.70 \mu \mathrm{mol} / \mathrm{L}$ [52]. Our re-analysis of the Bronx study showed that more than $40 \%$ of pregnant women have serum retinol $<0.70 \mu \mathrm{mol} / \mathrm{L}$, strongly suggesting that urgent actions need to be taken to reduce the VAD, especially in unusually susceptible ethnic groups, to reduce the risk of adverse health conditions of the mother [53] and diseases of offspring later in life [54,55]. Moreover, our results showed that genetic variations may be contributing to the VAD status differences between ethnic groups, at least in pregnant women. Further understanding of this association will ultimately enable adequate food interventions based on the genetic information that could be crucial to improve maternal vitamin $A$ status during pregnancy in these higher risk groups.

\section{Abbreviations}

eQTL: expression quantitative trait loci

GWAS: Genome-Wide Association Study

NHANES: National Health and Nutrition Examination Survey

PAGE: Population Architecture using Genomics and Epidemiology

VAD: vitamin A deficiency

\section{Declarations}

\section{Ethical Approval and Consent to Participate:}


We used publicly available data that no ethical approval is required.

\section{Consent for publication:}

Not applicable.

\section{Availability of data and materials:}

The serum retinol and demographic information of the Bronx dataset, originally published in the $J$ Perinat Med (PMID:30231012), are available by request. The National Health and Nutrition Examination Survey (NHANES) datasets were downloaded from the NHANES repository

(https://www.cdc.gov/nchs/nhanes/index.htm) and merged file in accordance with the NHANES guidelines and recommendations.

\section{Competing interests:}

All authors declare no competing interest.

\section{Funding:}

This work was supported by the National Institutes of Health under award number R01HL145302 (MS) and partially by NIH R01HD094778 and R01HD083331 (LQ). The content is solely the responsibility of the authors and does not necessarily represent the official views of the National Institutes of Health.

\section{Authors' Contributions:}

Wrote manuscript draft (MS,LQ), prepared illustrations (MS), approved final manuscript (MS, DG, TW, CRI, WC, JMG, LQ), conceived project (MS, LQ), analyzed data (MS), formulated research questions (MS, LQ), interpreted results (MS, TW, DG, CRI, JMG, WC, LQ), led investigation (MS). All authors read and approved the final manuscript.

\section{Acknowledgements:}

Not applicable.

\section{Authors' information:}

\section{Department of Genetics, Albert Einstein College of Medicine, Bronx, NY, USA}

John M. Greally and Masako Suzuki

Department of Epidemiology and Population Health, Albert Einstein College of Medicine, Bronx, NY, USA

Tao Wang and Carmen R. Isasi 
Department of Obstetrics and Gynecology and Women's Health, Stony Brook University Medical Center, Stony Brook, NY, USA

Diana Garretto

Department of Medicine, Columbia University, New York, NY USA

Wellington V. Cardoso

Department of Food Science and Rutgers Center for Lipid Research, and New Jersey Institute for Food, Nutrition, and Health, Rutgers University, New Brunswick, NJ, USA

Loredana Quadro

\section{References}

1. Vogel S, Piantedosi R, O'Byrne SM, Kako Y, Quadro L, Gottesman ME, et al. Retinol-binding proteindeficient mice: biochemical basis for impaired vision. Biochemistry. 2002;41:15360-8. doi:10.1021/bi0268551.

2. Quadro L, Blaner WS, Salchow DJ, Vogel S, Piantedosi R, Gouras $P$, et al. Impaired retinal function and vitamin A availability in mice lacking retinol-binding protein. EMBO J. 1999;18:4633-44. doi:10.1093/emboj/18.17.4633.

3. Liu X, Cui T, Li Y, Wang Y, Wang Q, Li X, et al. Vitamin A supplementation in early life enhances the intestinal immune response of rats with gestational vitamin A deficiency by increasing the number of immune cells. PLoS One. 2014;9:e114934. doi:10.1371/journal.pone.0114934.

4. Sirisinha S. The pleiotropic role of vitamin A in regulating mucosal immunity. Asian Pac J Allergy Immunol. 2015;33:71-89.

5. Williams JB, Napoli JL. Metabolism of retinoic acid and retinol during differentiation of F9 embryonal carcinoma cells. Proc Natl Acad Sci USA. 1985;82:4658-62. doi:10.1073/pnas.82.14.4658.

6. Lee TF, Mak KM, Rackovsky O, Lin Y-L, Kwong AJ, Loke JC, et al. Downregulation of hepatic stellate cell activation by retinol and palmitate mediated by adipose differentiation-related protein (ADRP). J Cell Physiol. 2010;223:648-57. doi:10.1002/jcp.22063.

7. Cammas L, Romand R, Fraulob V, Mura C, Dollé P. Expression of the murine retinol dehydrogenase 10 (Rdh10) gene correlates with many sites of retinoid signalling during embryogenesis and organ differentiation. Dev Dyn. 2007;236:2899-908. doi:10.1002/dvdy.21312.

8. Janick-Buckner D, Barua AB, Olson JA. Induction of HL-60 cell differentiation by water-soluble and nitrogen-containing conjugates of retinoic acid and retinol. FASEB J. 1991;5:320-5. doi:10.1096/fasebj.5.3.2001792.

9. Marx J, Naudé H, Pretorius E. The Effects of Hypo- and Hypervitaminosis a and Its Involvement in Foetal Nervous System Development and Post-Natal Sensorimotor Functioning - A Review. The British Journal of Development Disabilities. 2006;52:47-64. doi:10.1179/096979506799103677. 
10. Zile MH. Vitamin A and embryonic development: an overview. J Nutr. 1998;128 2 Suppl:455S-458S. doi:10.1093/jn/128.2.455S.

11. Tanumihardjo SA, Russell RM, Stephensen CB, Gannon BM, Craft NE, Haskell MJ, et al. Biomarkers of Nutrition for Development (BOND)-Vitamin A Review. J Nutr. 2016;146:1816S-48S. doi:10.3945/jn.115.229708.

12. https://www.cdc.gov/nutritionreport/pdf/4page_\%202nd\%20nutrition\%20report_508_032912.pdf. https://www.cdc.gov/nutritionreport/pdf/4page_\%202nd\%20nutrition\%20report_508_032912.pdf. Accessed 22 May 2020.

13. Hanson MA, Gluckman PD. Developmental origins of health and disease: new insights. Basic Clin Pharmacol Toxicol. 2008;102:90-3. doi:10.1111/j.1742-7843.2007.00186.x.

14. National Health and Nutrition Examination Surveys (NHANES) . Centers for Disease Control and Prevention (CDC). National Center for Health Statistics (NCHS). National Health and Nutrition Examination Survey Data. Hyattsville, MD: U.S. Department of Health and Human Services, Centers for Disease Control and Prevention. https://wwwn.cdc.gov/nchs/nhanes/Default.aspx. Accessed 13 Jul 2020.

15. Hanson C, Lyden E, Abresch C, Anderson-Berry A. Serum retinol concentrations, race, and socioeconomic status in of women of childbearing age in the united states. Nutrients. 2016;8. doi:10.3390/nu8080508.

16. Wirth JP, Petry N, Tanumihardjo SA, Rogers LM, McLean E, Greig A, et al. Vitamin A Supplementation Programs and Country-Level Evidence of Vitamin A Deficiency. Nutrients. 2017;9. doi:10.3390/nu9030190.

17. Garretto D, Kim Y-K, Quadro L, Rhodas RR, Pimentel V, Crnosija NA, et al. Vitamin A and $\beta$-carotene in pregnant and breastfeeding post-bariatric women in an urban population. J Perinat Med. 2019;47:183-9. doi:10.1515/jpm-2018-0142.

18. The National Health and Nutrition Examination Survey (NHANES).

19. de Pee S, Dary O. Biochemical indicators of vitamin A deficiency: serum retinol and serum retinol binding protein. J Nutr. 2002;132 9 Suppl:2895S-2901S. doi:10.1093/jn/132.9.2895S.

20. World Health Organization. Indicators for Assessing Vitamin A Deficiency and their Application in Monitoring and Evaluating Intervention Programmes. 1996. https://www.who.int/nutrition/publications/micronutrients/vitamin_a_deficiency/WHONUT96.10.pdf? ua=1. Accessed 13 Jul 2020.

21. Palmer AC, West KP, Dalmiya N, Schultink W. The use and interpretation of serum retinol distributions in evaluating the public health impact of vitamin A programmes. Public Health Nutr. 2012;15:120115. doi:10.1017/S1368980012000560.

22. Allele Frequency Aggregator. https://www.ncbi.nlm.nih.gov/snp/docs/gsr/alfa/. Accessed 13 Jul 2020.

23. Population Architecture using Genomics andEpidemiology. https://www.pagestudy.org/index.php/home. Accessed 13 Jul 2020. 
24. 1000 Genomes Project Consortium, Abecasis GR, Auton A, Brooks LD, DePristo MA, Durbin RM, et al. An integrated map of genetic variation from 1,092 human genomes. Nature. 2012;491:56-65. doi:10.1038/nature11632.

25. Sorlie PD, Avilés-Santa LM, Wassertheil-Smoller S, Kaplan RC, Daviglus ML, Giachello AL, et al. Design and implementation of the Hispanic Community Health Study/Study of Latinos. Ann Epidemiol. 2010;20:629-41. doi:10.1016/j.annepidem.2010.03.015.

26. GTEx Consortium. Human genomics. The Genotype-Tissue Expression (GTEx) pilot analysis: multitissue gene regulation in humans. Science. 2015;348:648-60. doi:10.1126/science.1262110.

27. R Foundation for Statistical Computing RCT. R: A Language and Environment for Statistical Computing. https://www.r-project.org/. Accessed 1 Nov 2020.

28. U.S. Census Bureau. US Census. https://data.census.gov/cedsci/. Accessed 13 Jul 2020.

29. Blumberg JB, Frei B, Fulgoni VL, Weaver CM, Zeisel SH. Contribution of dietary supplements to nutritional adequacy by socioeconomic subgroups in adults of the united states. Nutrients. 2017;10. doi:10.3390/nu10010004.

30. Gueguen S, Leroy P, Gueguen R, Siest G, Visvikis S, Herbeth B. Genetic and environmental contributions to serum retinol and alpha-tocopherol concentrations: the Stanislas Family Study. Am J Clin Nutr. 2005;81:1034-44. doi:10.1093/ajcn/81.5.1034.

31. The NHGRI-EBI Catalog of human genome-wide association studies. GWAS Catalog. https://www.ebi.ac.uk/gwas/. Accessed 13 Jul 2020.

32. Mondul AM, Yu K, Wheeler W, Zhang H, Weinstein SJ, Major JM, et al. Genome-wide association study of circulating retinol levels. Hum Mol Genet. 2011;20:4724-31. doi:10.1093/hmg/ddr387.

33. Oh DY, Talukdar S, Bae EJ, Imamura T, Morinaga H, Fan W, et al. GPR120 is an omega-3 fatty acid receptor mediating potent anti-inflammatory and insulin-sensitizing effects. Cell. 2010;142:687-98. doi:10.1016/j.cell.2010.07.041.

34. Hirasawa A, Tsumaya K, Awaji T, Katsuma S, Adachi T, Yamada M, et al. Free fatty acids regulate gut incretin glucagon-like peptide-1 secretion through GPR120. Nat Med. 2005;11:90-4. doi:10.1038/nm1168.

35. Oh DY, Walenta E. Omega-3 Fatty Acids and FFAR4. Front Endocrinol (Lausanne). 2014;5:115. doi:10.3389/fendo.2014.00115.

36. Quadro L, Hamberger L, Colantuoni V, Gottesman ME, Blaner WS. Understanding the physiological role of retinol-binding protein in vitamin A metabolism using transgenic and knockout mouse models. Mol Aspects Med. 2003;24:421-30. doi:10.1016/s0098-2997(03)00038-4.

37. Thompson SJ, Sargsyan A, Lee S-A, Yuen JJ, Cai J, Smalling R, et al. Hepatocytes are the principal source of circulating RBP4 in mice. Diabetes. 2017;66:58-63. doi:10.2337/db16-0286.

38. Pirazzi C, Valenti L, Motta BM, Pingitore P, Hedfalk K, Mancina RM, et al. PNPLA3 has retinyl-palmitate lipase activity in human hepatic stellate cells. Hum Mol Genet. 2014;23:4077-85. doi:10.1093/hmg/ddu121. 
39. Kovarova M, Königsrainer I, Königsrainer A, Machicao F, Häring H-U, Schleicher E, et al. The Genetic Variant I148M in PNPLA3 Is Associated With Increased Hepatic Retinyl-Palmitate Storage in Humans. J Clin Endocrinol Metab. 2015;100:E1568-74. doi:10.1210/jc.2015-2978.

40. Tian C, Stokowski RP, Kershenobich D, Ballinger DG, Hinds DA. Variant in PNPLA3 is associated with alcoholic liver disease. Nat Genet. 2010;42:21-3. doi:10.1038/ng.488.

41. He S, McPhaul C, Li JZ, Garuti R, Kinch L, Grishin NV, et al. A sequence variation (I148M) in PNPLA3 associated with nonalcoholic fatty liver disease disrupts triglyceride hydrolysis. J Biol Chem. 2010;285:6706-15. doi:10.1074/jbc.M109.064501.

42. Romeo S, Kozlitina J, Xing C, Pertsemlidis A, Cox D, Pennacchio LA, et al. Genetic variation in PNPLA3 confers susceptibility to nonalcoholic fatty liver disease. Nat Genet. 2008;40:1461-5. doi:10.1038/ng.257.

43. Graham TE, Yang Q, Blüher M, Hammarstedt A, Ciaraldi TP, Henry RR, et al. Retinol-binding protein 4 and insulin resistance in lean, obese, and diabetic subjects. N Engl J Med. 2006;354:2552-63. doi:10.1056/NEJMoa054862.

44. Manolescu D-C, Sima A, Bhat PV. All-trans retinoic acid lowers serum retinol-binding protein 4 concentrations and increases insulin sensitivity in diabetic mice. J Nutr. 2010;140:311-6. doi:10.3945/jn.109.115147.

45. Aeberli I, Biebinger R, Lehmann R, L'allemand D, Spinas GA, Zimmermann MB. Serum retinol-binding protein 4 concentration and its ratio to serum retinol are associated with obesity and metabolic syndrome components in children. J Clin Endocrinol Metab. 2007;92:4359-65. doi:10.1210/jc.20070468.

46. Jing L, Xiao M, Dong H, Lin J, Chen G, Ling W, et al. Serum Carotenoids Are Inversely Associated with RBP4 and Other Inflammatory Markers in Middle-Aged and Elderly Adults. Nutrients. 2018;10. doi: $10.3390 /$ nu10030260.

47. Zhou Z, Chen H, Ju H, Sun M. Circulating retinol binding protein 4 levels in nonalcoholic fatty liver disease: a systematic review and meta-analysis. Lipids Health Dis. 2017;16:180. doi:10.1186/s12944017-0566-7.

48. Blaner WS. Retinol-binding protein: the serum transport protein for vitamin A. Endocr Rev. 1989;10:308-16. doi:10.1210/edrv-10-3-308.

49. Borel P, Desmarchelier C. Genetic Variations Associated with Vitamin A Status and Vitamin A Bioavailability. Nutrients. 2017;9. doi:10.3390/nu9030246.

50. Looker AC, Johnson CL, Underwood BA. Serum retinol levels of persons aged 4-74 years from three Hispanic groups. Am J Clin Nutr. 1988;48:1490-6. doi:10.1093/ajcn/48.6.1490.

51. Centers for Disease Control and Prevention. Second Nutrition Report (2012). https://www.cdc.gov/nutritionreport/report_2012.html. Accessed 13 Jul 2020.

52. Vitamin A supplementation during pregnancy. https://www.who.int/elena/titles/guidance_summaries/vitamina_pregnancy/en/. Accessed 13 Jul 2020. 
53. Radhika MS, Bhaskaram P, Balakrishna N, Ramalakshmi BA, Devi S, Kumar BS. Effects of vitamin A deficiency during pregnancy on maternal and child health. BJOG. 2002;109:689-93. doi:10.1111/j.1471-0528.2002.01010.x.

54. Bhat PV, Manolescu D-C. Role of vitamin A in determining nephron mass and possible relationship to hypertension. J Nutr. 2008;138:1407-10. doi:10.1093/jn/138.8.1407.

55. Chen F, Marquez H, Kim Y-K, Qian J, Shao F, Fine A, et al. Prenatal retinoid deficiency leads to airway hyperresponsiveness in adult mice. J Clin Invest. 2014;124:801-11. doi:10.1172/JCl70291.

\section{Table 1}

Due to technical limitations Table 1 is available as a download in the Supplementary Files.

\section{Figures}

a ALFA rs10882272

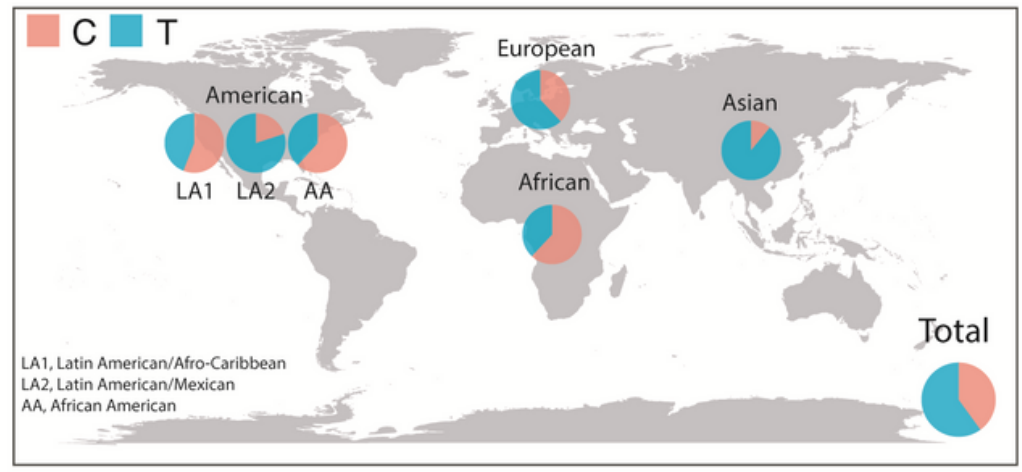

b

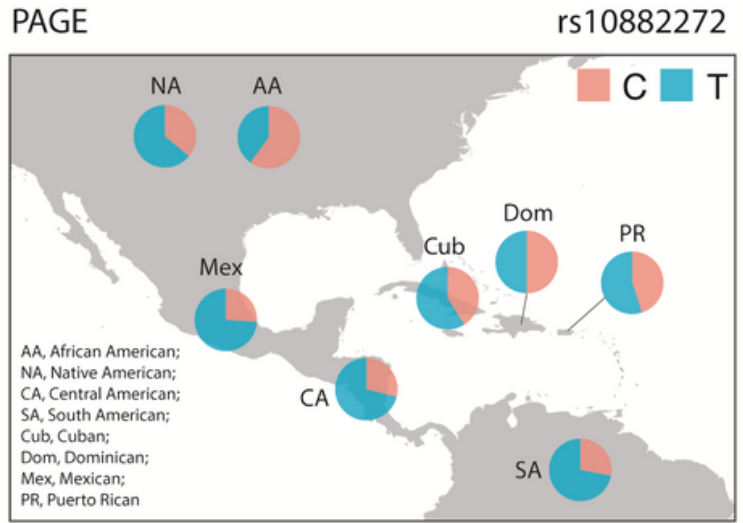

Figure 1

\section{Figure 1}

Variations of the allele frequencies of rs 10882272 We plotted the allele frequency of each ethnic group (a) Allele Frequency Aggregator (ALFA) and (b) Population Architecture using Genomics and Epidemiology (PAGE).

\section{Supplementary Files}

This is a list of supplementary files associated with this preprint. Click to download.

- Table1.xlsx

- Additionalfile1.pdf

- Additionalfile2.xlsx 